\title{
Qualidade dos registros de enfermagem em um hospital: auditoria
}

\author{
Quality of nursing records in a hospital: audit \\ Calidad de los registros de enfermería en un hospital: auditoria
}

Ludimila Cristina Souza Silva

ORCID: https://orcid.org/0000-0002-4342-217X

Faculdade Alfredo Nasser, Brasil

E-mail: professoraludimilacs@gmail.com

Marinésia Aparecida do Prado

ORCID: https://orcid.org/0000-0003-4652-0733

Universidade Federal de Goiás, Brasil

E-mail: marinesiaprado@gmail.com

Lilian Carla Carneiro

ORCID: https://orcid.org/0000-0003-4067-1506

Universidade Federal de Goiás, Brasil

E-mail: carlacarneriolilian@gmail.com

Aroldo Vieira de Moraes Filho

ORCID: https://orcid.org/0000-0003-2325-1374 Faculdade Alfredo Nasser, Brasil

E-mail: aroldodemoraes@gmail.com

Tatieny Aparecida Martins da Costa

ORCID: https://orcid.org/0000-0002-6265-4152 Faculdade Alfredo Nasser, Brasil

E-mail: tatieny.auditoria@gmail.com

Danielle Perdigão Oliveira e Ribeiro

ORCID: https://orcid.org/0000-0001-7404-5066 Faculdade Alfredo Nasser, Brasil

E-mail: daniperdigaooliveira@gmail.com

Ana Lucia Queiroz Bezerra

ORCID: https://orcid.org/0000-0002-3330-8859 Universidade Federal de Goiás, Brasil

E-mail: analuciaqueiroz@uol.com.br

Maria Alves Barbosa

ORCID: https://orcid.org/0000-0002-0861-9655 Universidade Federal de Goiás, Brasil

E-mail: maria.malves@gmail.com

\section{Resumo}

Objetivo avaliar por meio da auditoria a eficácia dos registros de enfermagem em um hospital público de Goiás. Método trata-se de um estudo descritivo e analítico, realizado em prontuários de pacientes hospitalizados em um hospital público de Goiás. Analisaram-se prontuários de pacientes com tempo de internação superior a cinco dias, em unidades de terapia intensiva neonatal, pediátrica e materna. Para análise dos dados, utilizou-se a comparação das médias das variáveis contínuas através do teste de Mann-Whitney. Resultado verificou-se que o preenchimento inadequado dos prontuários comprometeu os critérios da qualidade, antes e após a ação educativa. No entanto as médias inerentes às informações completas, incompletas e não preenchidas em relação aos prontuários foram significativamente reparadas após a ação educativa $(\mathrm{p}<0,05)$. As categorias, dados de identificação $(\mathrm{PP}=81,16 \%)$ e a execução das ordens médicas $(\mathrm{PP}=89,13 \%)$ apresentaram maiores índices de adequação quanto aos registros completos. A prescrição de enfermagem comprometeu a qualidade, quanto ao preenchimento incorreto dos registros em $(\mathrm{PP}=6,76 \%)$. Conclusão os registros de enfermagem não correspondem aos critérios da qualidade, mesmo após a ação educativa, tendo em vista que a eficácia dos registros passou por ajustes pequenos. Recomenda-se que ações educativas sejam instituídas nas políticas de saúde do hospital. Palavras-chave: Registros de enfermagem; Qualidade; Inconformidade; Intervenção; Controle; Prescrição.

\begin{abstract}
Objective to evaluate, through auditing, the effectiveness of nursing records in a public hospital in Goiás. Method This is a descriptive and analytical study, carried out on medical records of patients hospitalized in a public hospital in Goiás. hospital stay longer than five days in neonatal, pediatric and maternal intensive care units. For data analysis, we used the comparison of means of continuous variables through the Mann-Whitney test. Results it was verified that the inadequate filling of the medical records compromised the quality criteria, before and after the educational action. However, the means inherent to complete, incomplete and unfilled information in relation to medical records were significantly repaired after the educational action $(\mathrm{p}<0.05)$. The categories, identification data $(\mathrm{PP}=81.16 \%)$ and the
\end{abstract}


execution of medical orders $(\mathrm{PP}=89.13 \%)$ had higher adequacy rates regarding complete records. The nursing prescription compromised the quality, regarding the incorrect filling of the records in $(\mathrm{PP}=6.76 \%)$. Conclusion nursing records do not meet the quality criteria, even after the educational action, considering that the effectiveness of the records underwent minor adjustments. It is recommended that educational actions be instituted in the hospital's health policies.

Keywords: Nursing records; Quality; Non-compliance; Intervention; Control; Prescription.

\section{Resumen}

Objetivo evaluar, mediante auditoría, la efectividad de los registros de enfermería en un hospital público de Goiás. Método Se trata de un estudio descriptivo y analítico, realizado sobre historias clínicas de pacientes hospitalizados en un hospital público de Goiás. En unidades de cuidados intensivos neonatales, pediátricos y maternos. Para el análisis de los datos se utilizó la comparación de medias de variables continuas mediante la prueba de Mann-Whitney. Resultados Se verificó que el llenado inadecuado de las historias clínicas comprometió los criterios de calidad, antes y después de la acción educativa. Sin embargo, los medios inherentes a la información completa, incompleta y sin completar en relación a la historia clínica se repararon significativamente después de la acción educativa $(\mathrm{p}<0.05)$. Las categorías, datos de identificación ( $\mathrm{PP}=81,16 \%$ ) y ejecución de órdenes médicas $(\mathrm{PP}=89,13 \%$ ) tuvieron mayores tasas de adecuación con respecto a registros completos. La prescripción de enfermería comprometió la calidad, con respecto al llenado incorrecto de los registros en ( $\mathrm{PP}=6,76 \%$ ). Conclusión Los registros de enfermería no cumplen con los criterios de calidad, incluso después de la acción educativa, considerando que la efectividad de los registros sufrió pequeños ajustes. Se recomienda que se instituyan acciones educativas en las políticas de salud del hospital.

Palabras clave: Registros de enfermería; Calidad; Incumplimiento; Intervención; Control; Prescripción.

\section{Introdução}

O impacto das transformações a partir da globalização e do desenvolvimento tecnológico na saúde, resultou na necessidade de reestruturar o processo de trabalho com o intuito de garantir a eficácia da assistência. Diante disso tornou-se necessário avaliar constantemente o serviço de saúde com o intuito de garantir uma assistência de baixo custo e de excelência (Camelo, Pinheiro, Campos \& Oliveira, 2009).

Nessa perspectiva, o processo de avaliação em saúde tornou-se frequente e têm sido utilizado para medir a efetividade das ações programáticas, os custos, evitar ou reduzir os incidentes ocasionados por eventos adversos às pessoas na ambiência de saúde (World Health Organization, 2009).

Para o processo de avaliação em saúde, os registros em prontuários configuram-se como um importante documento para mensurar qualidade da assistência e os custos dela decorrentes. Estes devem ser realizados de forma clara, completa e consistente, uma vez que os registros possibilitam a comunicação entre os profissionais, favorecendo a continuidade do cuidado e o respaldo das ações assistenciais, junto ao paciente e à família (Cordeiro, Dyniewicz \& Kalinowski, 2011).

Os registros podem subsidiar ainda ações jurídicas, de pesquisa e ensino, além de ser um importante indicador de qualidade (Barbosa \& Trochin, 2015). Portanto o ato de registrar as ações realizadas pela equipe de saúde facilita a comprovação da assistência implementada e os aspectos ético-legal exigidos e controla os desperdícios com atribuídos à assistência em saúde (Mazieiro, 2013).

Com relação à obrigatoriedade dos registros, a Resolução do Conselho Federal de Enfermagem (COFEN)191/96 determina a importância da qualidade das anotações de enfermagem, haja vista que estas, podem ser utilizadas em processos laborais, tanto para acusar como para defender os trabalhadores profissionais e a instituição, mediante investigações motivadas por inadequações relacionadas ao processo assistencial (COFEN, 1986). Desse modo, o registro é de caráter formal e vital para revelar indicadores que reforçam uma assistência em conformidade as diretrizes vigentes (Seignemartin, Jesus, Vergílio \& Silva, 2013).

A existência de normas que fundamentam a realização dos registros, a complexidade do processo assistencial e a evolução tecnológica em saúde, tem impactado nos custos e na melhoria contínua da qualidade da assistência. Essa impactação tem exigido trabalhadores qualificados, sendo a sua participação em ações de educação continuada, de fundamental importância. 
Fato que traz como objetivo despertar estes profissionais para uma prática segura e uma assistência de qualidade em saúde (Barbosa, eiras, Leão \& Barnabé, 2015).

Em contrapartida, a ausência dos registros em prontuários ou realizados de forma inadequada, indicam uma assistência descomprometida com a segurança do paciente, além da dificultar o processo de avaliação em saúde (Mazieiro, 2013). A presença de registros em desacordo com os critérios da eficácia têm impacto negativo sobre a comunicação entre a equipe de saúde, a continuidade e a integralidade das ações assistenciais e a tomada de condutas em relação à terapêutica do paciente (Borges, Azevedo, Amorim, Figueiredo \& Ribeiro, 2017).

Apesar da importância dos registros em prontuários e seus desdobramentos, as evidências científicas aliadas a vivência clínica na ambiência em saúde, têm sinalizado lacunas quanto a eficácia desses registros. Portanto essa problemática torna-se ainda mais evidente pelo seu impacto nos custos e na qualidade do serviço de saúde. Tais lacunas sinalizam a necessidade de estudos nessa temática, com o intuito de subsidiar estratégias capazes de impulsionar políticas de saúde valorizando tais fragilidades em relação à sensibilização dos trabalhadores de saúde ao ato de registrar suas ações, cumprindo as exigências ético e legais da profissão.

Este estudo teve como objetivo avaliar por meio da auditoria a eficácia dos registros de enfermagem em um hospital público de Goiás.

\section{Metodologia}

Estudo descritivo e analítico, realizado por meio da auditoria retrospectiva e prospectiva sobre a eficácia dos registros de enfermagem em uma instituição especializada em saúde materna e infantil estadual, integrada ao Sistema Único de Saúde de Goiás.

Coletou-se registros de 45 prontuários de pacientes com tempo de internação superior a cindo dias, em unidades de terapia intensiva neonatal, pediátrica e materna, em dois momentos distintos. A coleta foi realizada em dois momentos distintos, sendo o primeiro de 25 de agosto a 10 de setembro de 2016, contemplando 22 prontuários. Após a análise das informações, ofereceu-se uma ação educativa aos trabalhadores de saúde da instituição, abordando aspectos científicos, éticos e legais no contexto da assistência segura e de qualidade. O segundo ocorreu de 25 de abril a 10 de maio de 2017, considerando 23 prontuários.

A diferença de leitos entre o primeiro e o segundo momento da coleta dos dados se deu pelo fato no primeiro os dez leitos da UTI neonatal e pediátrica permaneciam ocupados, e apenas dois da materna. Já no segundo a UTI materna apresentava três leitos ocupados, totalizando 23 prontuários.

Propôs-se um intervalo de três meses, entre as coletas, para avaliar a eficácia dos registros após a capacitação dos trabalhadores. Foram incluídos todos os prontuários de pacientes que estavam hospitalizados nas Unidades de Terapia Intensiva Neonatal, Pediátrica e Materna, tempo de internação $\geq$ a cinco dias. Foram excluídos os prontuários de pacientes com tempo de internação $\leq$ a cinco dias, de acordo com o instrumento de coleta de dados proposto por Haddad (Haddad, 2004).

As variáveis primárias constituíram-se sexo, idade, diagnóstico e período de internação. As secundárias foram, prescrição, procedimentos e anotações de enfermagem, execução de ordens médicas, condições de alta, critérios específicos da UTI materna, pediátrica e neonatal.

A qualidade dos registros nos prontuários, seguiu os níveis de avaliação que variaram de 1 a 5 , classificados como: 1 não se aplica, 2 - completo, 3 - incompleto, 4 - não preenchido e 5 -incorreto (Mazieiro, 2013). Os dados foram coletados pela pesquisadora, visando assegurar a qualidade das informações.

A ação educativa foi desenvolvida na própria instituição, nos dias 15 e 20 de dezembro de 2016, com carga horária total de oito horas. Foram disponibilizadas 40 vagas, em decorrência da capacidade máxima do espaço físico destinado ao evento na 
instituição para facilitar a participação dos profissionais. Participaram 33 profissionais de saúde, que não cumpriam escala de trabalho, tendo em vista que a instituição não liberou os trabalhadores escalados para participarem do evento.

Como forma de motivar a participação dos trabalhadores, foram oferecidos certificados, material didático, lanche e sorteio de brindes.

A organização da atividade educativa ocorreu de forma conjunta entre a comissão organizadora do evento, composta por docentes e discentes do programa de pós-graduação e gestores de enfermagem do hospital.

A ação educativa ocorreu em dois módulos:

No primeiro módulo contextualizou-se os aspectos científicos, éticos e legais no contexto da assistência segura e de qualidade, a importância da qualificação profissional, tomada de decisão, exercício legal da profissão, prestação do cuidado e o registro da assistência de enfermagem no prontuário (conceito, organização e finalidade). Reforçou ainda o prontuário como fontes de informação para auditoria interna (conceito, finalidade e tipos de auditoria) e de avaliação da qualidade da assistência em saúde.

No segundo módulo oferecido cinco dias após o primeiro, em decorrência da disponibilidade do espaço físico destinado a atividade educativa na instituição. Nesse módulo abordou-se, questões referentes à auditoria interna como ferramenta da qualidade da assistência, a responsabilidade do trabalhador de saúde frente aos registros nos prontuários e à otimização dos custos. Apresentou-se ainda o PDCA como proposta para melhoria da qualidade do serviço de saúde.

O projeto foi aprovado em Comitê de Ética em Pesquisa, sob o protocolo CEPESH/HMI/GO, nº 1.447.390, por se tratar de dados secundários, utilizando-se dos prontuários, liberado pelo CEPESH/HMI/GO o Termo de Consentimento Livre e Esclarecido.

A confidencialidade dos prontuários foi assegurada utilizando-se de códigos do tipo 01, 02, 03 e assim sucessivamente, para identificar o prontuário do paciente, seguido da letra "A" para as análises efetuadas antes da realização da ação educativa, e da letra "B" para as análises posteriores à ação educativa, acrescidos das abreviações "NEO" para pacientes hospitalizados na unidade de terapia intensiva neonatal, "PED" para pacientes hospitalizados na unidade de terapia intensiva pediátrica e "mat" para unidade de terapia intensiva materna. Exemplo: 01ANEO.

Os dados relativos a avaliação da qualidade dos registros em prontuários foram organizados em planilha Microsoft Office/Excel e em seguida, exportados para o programa de análise estatística o software STATA ${ }^{\circledR}$ versão 12.0.

Foi realizada análise descritiva dos dados categóricos que foram apresentados em frequências absolutas e relativas e para as variáveis contínuas médias e desvio padrão. A análise estatística segmentou-se na amostra dos prontuários antes e após a ação educativa. Para as informações obtidas em cada questionário, foram estimadas as porcentagens das informações corretas, incompletas, não preenchidas e incorretas para comparação.

Utilizou-se o teste de qui-quadrado e exato de Fisher para testar a homogeneidade dos grupos em relação às proporções. Foi realizado o teste de normalidade Shapiro-Wilk que retornou os resultados positivos e negativos e, dessa forma, optou-se por usar estatística não paramétrica para comparação das médias das variáveis contínuas, especificamente o teste de Mann-Whitney. O nível de significância utilizado para os testes foi de 5\%. Foi utilizado o software STATA $^{\circledR}$ versão 12.0, nesta análise.

Ao realizar o cálculo de amostragem à posteriori para teste de qui-quadrado (tabelas de contingência), considerou-se um efeito de delineamento de 1,10 (obtido de dados de frequências relativas da variável), "Os dados de identificação do paciente estão corretos?", por ser a primeira variável significativa do estudo. Considerou-se um alfa de 0,05 e um nível de significância de 5\% para uma amostra de 45 indivíduos e obteve-se como resultado o valor de poder do teste de aproximadamente $100 \%$, favorável ao uso do teste estatístico realizado nesse estudo. O cálculo foi realizado pelo programa $G^{*}$ Power 3.1.9.2 ${ }^{\circledR}$. 
Consideraram-se bons resultados quando a análise dos prontuários indicava que, pelo menos, $80 \%$ das prescrições de enfermagem foram executadas completamente; até $15 \%$ para preenchimento incompleto; menor que $5 \%$ para prescrições não preenchidas e 0\% para elaboração incorreta (Haddad, 2004; Cianciarullo, Fugulin \& Andreoni, 1998).

\section{Resultados}

O preenchimento dos prontuários analisados foi considerado inadequado antes e após a intervenção educativa. Contudo, as médias das porcentagens referentes aos registros completos foram significativamente maiores após a intervenção, consequentemente, as médias de informações incompletas e não preenchidas também foram menores $(\mathrm{p}<0,05)$. Por outro lado, a média das porcentagens de informações preenchidas incorretamente foi maior após a intervenção ( $\mathrm{p}=0,027)$ (Tabela 1).

Tabela 1. Qualidade dos registros em prontuários de pacientes hospitalizados em uma instituição de saúde de Goiás, antes e após ação educativa. Goiânia-Go. 2017.

\begin{tabular}{llll}
\hline Registros nos prontuários & Antes N=22 & Depois N=23 & P-valor* \\
& Média (DP) & Média (DP) & \\
\hline & & & \\
Completos & $35,47(12,84)$ & $66,70(17,98)$ & $<\mathbf{0 , 0 0 1}$ \\
Incompletos & $44,23(14,46)$ & $19,28(11,79)$ & $<\mathbf{0 , 0 0 1}$ \\
Não preenchidos & $10,92(6,60)$ & $5,30(5,38)$ & $<\mathbf{0 , 0 0 1}$ \\
Incorretos & $0,86(1,67)$ & $1,68(2,82)$ & $\mathbf{0 , 0 2 7}$ \\
\hline
\end{tabular}

*p-valor- teste de Mann-Whitney com nível de significância de 5\%. Fonte: Autores.

A Tabela 2 apresenta o percentual de preenchimento (PP) completo, incompleto, não preenchido e incorreto dos registros, avaliados antes e após a intervenção educativa.

Tabela 2. Percentual dos registros em prontuários de pacientes hospitalizados em uma instituição de saúde de Goiás, antes e após ação educativa. Goiânia-Go. 2017.

\begin{tabular}{|c|c|c|c|c|c|c|c|c|}
\hline \multirow[t]{2}{*}{$\begin{array}{l}\text { Categorias } \\
\text { avaliadas }\end{array}$} & \multicolumn{2}{|c|}{ Completo } & \multicolumn{2}{|c|}{ Incompleto } & \multicolumn{2}{|c|}{$\begin{array}{l}\text { Não } \\
\text { preenchido }\end{array}$} & \multicolumn{2}{|c|}{ Incorreto } \\
\hline & $\begin{array}{l}\text { Antes } \\
(\%)\end{array}$ & $\begin{array}{l}\text { Após } \\
(\%)\end{array}$ & $\begin{array}{l}\text { Antes } \\
(\%)\end{array}$ & $\begin{array}{l}\text { Após } \\
(\%)\end{array}$ & $\begin{array}{l}\text { Antes } \\
(\%)\end{array}$ & $\begin{array}{l}\text { Após } \\
(\%)\end{array}$ & $\begin{array}{l}\text { Antes } \\
(\%)\end{array}$ & $\begin{array}{l}\text { Após } \\
(\%)\end{array}$ \\
\hline A - dados de identificação & 40,91 & 81,16 & 59,10 & 18,84 & 0 & 0 & 0 & 0 \\
\hline B - Prescrição de enfermagem & 22,73 & 51,21 & 61,62 & 39,61 & 14,65 & 2,42 & 1,00 & 6,76 \\
\hline $\begin{array}{l}\mathrm{C}-\text { Procedimentos de } \\
\text { enfermagem }\end{array}$ & 41,26 & 53,51 & 22,38 & 21,07 & 18,18 & 6,69 & 4,19 & 3,34 \\
\hline D - Anotações de enfermagem & 17,13 & 47,49 & 43,36 & 20,40 & 15,38 & 9,70 & 0 & 0 \\
\hline $\begin{array}{l}\mathrm{E}-\text { Execução de ordens } \\
\text { médicas }\end{array}$ & 51,14 & 89,13 & 39,77 & 10,87 & 7,95 & 0 & 0 & 0 \\
\hline $\begin{array}{l}\mathrm{F}-\mathrm{UTI} \text { pediátrica, neonatal e } \\
\text { materna }\end{array}$ & 39,67 & 77,72 & 39,20 & 4,90 & 9,66 & 13,0 & 0 & 0 \\
\hline Total geral da prescrição & 32,17 & 59,91 & 41,18 & 20,70 & 13,54 & 6,79 & 1,27 & 2,09 \\
\hline
\end{tabular}

Fonte: Autores. 
Ao avaliar o conjunto de categorias antes e após a intervenção educativa, observa-se que não foi atingido o nível e qualidade preconizado, porém, os resultados sinalizam uma melhora significativa na qualidade dos registros em prontuários apresentando uma elevação na frequência de aproximadamente 50\% quando comparados aos dados do primeiro momento. Por outro lado, houve piora na porcentagem geral quanto ao preenchimento incorreto dos registros ( $\mathrm{PP}=2,09 \%)$.

Ao analisar isoladamente cada categoria percebe-se que duas categorias indicaram que, pelo menos, $80 \%$ das prescrições foram preenchidas completamente. Duas categorias até $15 \%$ de preenchimento incompleto. Quanto ao não preenchimento das prescrições de enfermagem, três categorias apresentaram frequência menor de 5\%, e quatro apresentaram índice $0 \%$ de preenchimento incorreto das mesmas.

A execução das ordens médicas nas UTI neonatal, pediátrica e materna atingiram níveis inferiores a $15 \%$ de preenchimento incompleto, portanto essas categorias estão adequadas aos parâmetros considerados satisfatórios.

Quanto ao não preenchimento, as categorias que apresentaram incidência inferior a 5\% referiram-se aos dados de identificação, prescrição de enfermagem e execução de ordens médicas. As categorias que apresentaram $0 \%$ de preenchimento incorreto foram, dados de identificação, anotações de enfermagem, execuções de ordens médicas e UTI neonatal, pediátrica e materna.

A categoria prescrição de enfermagem observou-se melhora quanto aos registros completos após a intervenção, mas por outro lado houve piora quanto aos registros incorretos. Quatro itens avaliados nessa categoria apresentaram elevado percentual de preenchimento "Incompleto", como por exemplo, os registros da admissão do paciente, pois esse permite conhecer as condições gerais do paciente ( $\mathrm{PP}=47,83 \%)$; a prescrição indica grau de dependência do paciente ( $\mathrm{PP}=78,26 \%)$; a prescrição indica ações referentes aos sinais e sintomas pertinentes à patologia e evolução do paciente ( $\mathrm{PP}=56,52 \%)$; os procedimentos foram preenchidos, quanto às características do paciente e às necessidades de sua patologia $(\mathrm{PP}=100 \%)$. Um item apresentou elevado percentual de preenchimento "Incorreto": a primeira prescrição revela a prioridade de atendimento ( $\mathrm{PP}=60,87 \%)$.

$\mathrm{Na}$ categoria procedimentos de enfermagem, dois itens apresentaram elevado PP "Incompleto": há registros de higiene oral pelo menos três vezes ao dia ( $\mathrm{PP}=100 \%$ ); há registros de higiene corporal diária ( $\mathrm{PP}=65,22 \%)$. Quanto ao "Não Preenchido" um item destacou-se: há registros de episódios eméticos ( $\mathrm{PP}=86,96 \%)$. O item procedimentos invasivos registrados e datados, sinalizou um elevado percentual de preenchimento "Incorreto" ( $\mathrm{PP}=43,48 \%)$. Os registros dos sinais vitais, fezes e urina foram significativamente maiores após a intervenção $(p<0,05)$.

Dois itens da categoria anotações de enfermagem apresentaram um índice elevado de preenchimento "Incompleto": há pelo menos uma anotação descritiva em cada plantão ( $\mathrm{PP}=52,17 \%)$; há horário e rubrica em cada anotação ( $\mathrm{PP}=73,91 \%)$. Dois itens apresentaram destacaram-se pela porcentagem elevada de "Não Preenchido": prescrição indica ações relacionadas ao atendimento de necessidades psicobiológicas $(\mathrm{PP}=69,57 \%)$; as anotações indicam as condições emocionais do paciente $(\mathrm{PP}=56,52 \%)$.

A execução de ordens médicas apresentou melhora quanto aos registros completos ( $\mathrm{PP}=89,13 \%)$ após a intervenção educativa. A categoria unidades de terapia intensiva neonatal, pediátrica e materna também apresentou melhora quanto aos registros completos $(\mathrm{PP}=77,72 \%)$ e apenas o item relativo há registros de mudança de decúbito programada apresentou índice de registro "Não Preenchido" elevado (PP=100\%).

Nos dados de identificação avaliou-se o preenchimento correto dos dados de identificação do paciente; preenchimento completo dos dados de identificação do paciente; registro de data, hora, $n^{\circ}$ do COREN (Conselho Regional de Enfermagem) e assinatura do enfermeiro na prescrição de enfermagem.

A identificação dos dados do paciente de forma correta e completa foi, significativamente, maior após a intervenção $(\mathrm{p}<0,05)$. Por outro lado, a média das informações referente ao registro de data, hora, $\mathrm{n}^{\circ}$ do COREN e assinatura do enfermeiro preenchida de forma incompleta foi maior após a educação educativa. 


\section{Discussão}

Estudos confirmam que a qualidade e o preenchimento dos registros nos prontuários de forma completa, clara e objetiva são de grande relevância para a assistência em saúde, pois, a comunicação escrita em conformidade com a legislação vigente, promove uma assistência sistematizada, qualificada e efetiva (Barreto, Azevedo, Amorim, Figueiredo \& Ribeiro, 2017).

A análise da qualidade dos registros no presente estudo mostrou melhora quanto ao preenchimento completo dos registros nos prontuários, o que sinalizar um pequeno movimento dos profissionais em relação a qualidade dos registros nos prontuários.

A necessidade de registrar as ações de enfermagem envolve aspectos legais da profissão e isso encontra-se justificado pela Resolução do Conselho Federal de enfermagem/358/09, que diz que "a execução do processo de enfermagem deve ser registrada formalmente" (COFEN, 2009).

É relevante que o profissional compreenda que os registros nos prontuários fazem parte das atribuições legais da profissão. Registrar de forma clara, completa e consistente é uma ação obrigatória e não voluntária, pois formaliza as ações do processo de trabalho da enfermagem, com a finalidade de respaldar o profissional e o paciente em caso de intercorrências envolvendo a assistência em saúde.

Os registros nos prontuários devem ser realizados em conformidade com a legislação vigente, pois as informações registradas permitem o respaldo ético e legal do profissional em casos de danos ao paciente (Morais, Batista, Castro, Assunção \& Castro, 2015).

O preenchimento inadequado de dados como número do registro no Conselho Regional de Enfermagem (COREN) e a assinatura do profissional que executou a assistência mostrou-se insatisfatório, o que infringe o Código de Ética Profissional. Para evitar esse tipo de inconformidade existem normativas como a Resolução Cofen n⿳0 311/07 que determina que o profissional de enfermagem, por questão de ética, deve prestar informações completas e fidedignas referentes ao processo de cuidar da pessoa. Assim como, o dever de registrá-las nos prontuários e documentos próprios da enfermagem, além de colocar o número de registro no COREN e assinatura identificando quem executou o procedimento (COFEN, 2007).

As inconformidades, apresentadas pelas anotações sejam elas associadas aos dados de identificação incompletos ou ausentes, implicam na desestruturação do processo de cuidar em saúde, na fragilidade da comunicação entre os profissionais e no comprometimento da continuidade e integralidade da práxis segura (Barreto, Lima \& Xavier, 2016).

Os dados sobre o procedimento, checagem, evolução e a identificação do executor da atividade podem ser considerados indicadores de maior índice de divergência e, por isso, responsáveis por determinadas glosas. De acordo com a literatura a ausência dessas informações pode significar para a equipe de auditoria a não realização do procedimento e, dessa forma, prejudicar financeiramente a instituição, que provavelmente não receberá pelo procedimento realizado, em decorrência da inconformidade dos registros de enfermagem (Barreto, Lima \& Xavier, 2016).

Outra inconformidade que compromete a qualidade da assistência é a incoerência entre a prescrição e a evolução de enfermagem, uma vez que essa situação fragiliza o processo de assistência de enfermagem, impedindo a continuidade do cuidado de forma segura e com qualidade. Achados na literatura têm mostrado que em algumas situações, os registros da assistência implementada não são relacionados com a prescrição de enfermagem, não atendem às necessidades do paciente, ou ainda, não correspondem ao quadro e à patologia do paciente (Diniz, Silva, Figueiredo \& Tonini, 2015).

Além dessas inconformidades, a ausência de registros de procedimentos e orientações impedem que a assistência seja validada, o que poderá implicar em questões etico-legais. Por isso as evidências científicas afirmam que registrar e padronizar os procedimentos de enfermagem são ações de impacto para autenticar e aperfeiçoar o processo de trabalho em saúde (Colaço et al., 2015) 
$\mathrm{O}$ ato de registrar permite que as informações existentes no prontuário tornem-se acessíveis a toda a equipe na tomada de decisão e na prática, porém a partir da análise dos dados observou-se uma fragilidade nesse contexto, principalmente quanto à rasuras, letra ilegível, ausência ou inexistência de registros.

A literatura corrobora com esses achados, pois em outros estudos parte significativa das orientações e encaminhamentos realizados pela enfermagem também não foram registradas, deixando, portanto, de evidenciar a complexidade e a importância da assistência implementada (Diniz, Silva, Figueiredo \& Tonini, 2015). Portanto, vale ressaltar que registros em conformidade podem minimizar as perdas econômicas institucionais, fortalecer a enfermagem como ciência, e ainda respaldar juridicamente o profissional e o paciente.

Diante disso observa-se que evoluções, condutas e prescrições de enfermagem devem sempre ser registradas de forma criteriosa. O estudo mostrou ainda que alguns registros de enfermagem apresentavam-se semelhantes às dos registros dos médicos, e isso sinaliza uma fragilidade preocupante quanto à eficácia dos registros de enfermagem.

Evidências na literatura associam tal situação à ausência de leitura sistemática e rotineira das prescrições como instrumento de trabalho, dessa forma é necessário despertar nos profissionais de enfermagem a necessidade de reconhecer a importância da elaboração dos registros e da implantação da Sistematização da Assistência de Enfermagem (SAE). A SAE tratase de uma estratégia de intervenção da assistência de enfermagem, visando à melhoria da qualidade da assistência (Claudino, Gouveia, Santos \& Lopes, 2013).

A Sistematização da Assistência de Enfermagem (SAE) apresenta-se como um instrumento para agregar qualidade ao cuidado oferecido aos pacientes e por isso acredita-se que a construção de softwares assistenciais fundamentados nas etapas da SAE seja uma importante estratégia para contemplar as necessidades de seus clientes (Wiley, Steffens, Berry \& Leasky, 2017).

O gerenciamento da assistência através de softwares permite ao profissional, atuar in loco e desenvolver a maioria de suas atividades no próprio computador, incentivando o desenvolvimento de habilidades científicas e competências da equipe, oferecendo maior segurança e comunicação durante o processo assistencial (Claudino, Gouveia, Santos \& Lopes, 2013)

As inconformidades apresentadas pela falta de uma assistência sistematizada podem gerar consequências e desconforto para o paciente, por isso o processo de avaliação dos cuidados prestados apresenta-se como uma forma de prevenir possíveis riscos ou prejuízos aos pacientes (Instefjord, Askjaer, Espehaug \& Graverholt, 2014). Para alcançar maior impacto na qualidade, é importante que todos os profissionais envolvidos na assistência estejam comprometidos com processo de melhorias, a partir das orientações e educação realizadas pelo serviço.

Acredita-se que os registros nos prontuários dos pacientes deverão ser monitorados por meio da auditoria interna, com vistas a melhorar tais indicadores que deverão ser avaliados periodicamente e discutidos com a equipe multidisciplinar.

A partir dessa estratégia é possível identificar a necessidade de capacitação da equipe quanto aos registros, habilidades e competências profissionais, como parte da política de saúde e organizacional da instituição, focada na melhoria da qualidade dos registros, e do processo assistencial em toda sua complexidade assistencial (Borges, Azevedo, Amorim, Figueiredo \& Ribeiro, 2017).

\section{Conclusão}

A avaliação da qualidade dos registros de enfermagem por meio da auditoria, e de uma ação educativa, sinalizou melhora significativa na eficácia dos registros de enfermagem.

Ações como o planejamento das atividades, ações educativas, treinamentos sistematizados, padronização da assistência e capacitação da equipe para a valorização da eficácia dos registros, podem ser consideradas como um caminho para uma gestão organizacional eficaz e comprometida com a qualidade dos cuidados. 
Porém, acredita-se que o monitoramento dos registros deve ser incorporado a política de saúde da instituição, assim como, um programa de educação permanente com o objetivo de qualificar periodicamente seus trabalhadores.

Recomenda-se como estratégias o uso da auditoria interna, como ferramenta de avaliação dos registros visando à qualidade da assistência em saúde em conformidade aos princípios de segurança do Sistema único de Saúde.

Outra recomendação, diz respeito à implementação de protocolos assistenciais com o objetivo gerencial, educacional e de comunicação, corroborando com o aprimoramento das habilidades e competências dos profissionais, em relação ao processo de trabalho, no ambiente laboral, em especial as unidades de terapia intensiva.

\section{Referências}

Camelo, S. H. H., Pinheiro, A., Campos, D., \& Oliveira, T. L. (2009). Auditoria de enfermagem e a qualidade da assistência à saúde: uma revisão da literatura. Rev Eletr Enf. 2009; 11(4): 1018-25.

World Health Organization. (WHO). (2009). The conceptual framework forctheinternationalclassification for patientsafety. Final Technical Report. Chapter The InternationalClassification for PatientSafety. Key ConceptsandPreferredTerms [Internet]. Geneva: WHO; 2009.

Cordeiro, F. F. , Dyniewicz, A. M., \& Kalinowski, L .C. (2011) Auditorship in nursing records at an intensive care unit. Rev enferm UFPE on line. 2011; 5(5): 1187-192.

Barbosa S F, \& Trochin D M R. (2015). Manual for monitoring the quality of nursing home care records. Rev Bras Enferm. 2015; 68 (2): 253-60.

Mazieiro V G. (2013). Qualidade dos Registros dos Controles de Enfermagem em um hospital universitário. Rev Min Enferm. 2013; 17 (1):165-170.

Conselho Federal de Enfermagem. COFEN. (1986). Dispõe sobre a regulamentação do exercício da Enfermagem e dá outras providências.Lei nº7.498/86, de 25 de junho de 1986 .

Seignemartin B A, Jesus L R, Vergílio M S T G, \& Silva E M S. (2013). Avaliação da qualidade das anotações de enfermagem no pronto atendimento de um hospital escola. Rev Rene. 2013; 14 (6): 1123-32.

Barbosa A P, Eiras F C, Leão E C, \& Barnabé A S. (2015). Organização de processos na melhoria da qualidade de registros assistenciais de enfermagem. Ver Raunp. 2015; 2 (2):121-130.

Borges F F D, Azevedo C T, Amorim T V, Figueiredo M A G, \& Ribeiro R G M. (2017).Importância das anotações de enfermagem segundo a equipe de enfermagem: implicações profissionais e institucionais. Revista de Enfermagem do Centro-Oeste Mineiro. $2017 ; 1147$ - 1154.

Haddad M C L. (2004). Qualidade da assistência de enfermagem: o processo de avaliação em hospital universitário público. Tese (Doutorado em Enfermagem) - Escola de Enfermagem de Ribeirão Preto da Universidade de São Paulo. Ribeirão Preto, 2004. $201 f$.

Cianciarullo T I, Fugulin F M T, \& Andreoni S. C. Q (1998). A hemodiálise em questão - opção pela qualidade da assistência. Ícone, 1998.

Barreto J A, Lima G G, \& Xavier C F. (2016). Inconsistências das Anotações de Enfermagem no Processo de Auditoria. Rev Cent O Min. 2016; 1 (6): 20812093 .

Conselho Federal de Enfermagem (COFEN). (2017). Resolução 358/2009, de 15 de Outubro de 2009. Dispõe sobre a Sistematização da Assistência de Enfermagem e a implementação do Processo de Enfermagem em ambientes, públicos ou privados. Brasília, 2009. http://www.cofen.gov.br/resoluo-cofen3582009 4384.html.

Morais C G X, Batista S E M, Castro J F L, Assunção S S, \& Castro G M O. (2015). Registros de enfermagem em prontuário e suas implicações na qualidade assistencial segundo os padrões de acreditação hospitalar: um novo olhar da auditoria. Ver Acred. 2015 ; 5 (9) 64-84.

Saranto K, Kinnunen UM, Kivekas E, Lappalainen A M, Liljamo P, \& Rajalahti E. (2014). Impacts of structuring nursing records: a systematic review. Scan J Caring Sci. 2014; 28: 629-647.

Conselho Federal de Enfermagem (COFEN).(2017). Resolução 311/2007, de 08 de fevereiro de 2007. Aprova a Reformulação do Código de Ética dos Profissionais de Enfermagem. Rio de Janeiro, 2007. Disponível em: http://www.cofen.gov.br/wp-content/uploads/2012/03/resolucao_311_anexo.pdf. Acesso em: 27/08/2017.

Diniz S O S, Silva O S, Figueiredo N M A, \& Tonini T. (2015). Qualidade dos registros de enfermagem: reflexões analíticas em suas formas e conteúdos. Rev Enferm. UFPE on line. 2015; 9 (10): 9616-23.

Claudino H G, Gouveia E M L, Santos S R, \& Lopes M E L . (2013). Auditoria em registros de enfermagem: revisão integrativa da literatura. Rev. Enferm. 2013; 21 (3): 397-402.

Colaço A, Rozado M F, Nascimento E R P, Delacanal L D, Dair BA, \& Walnice J.. (2015)Registro da avaliação de enfermagem em terapia intensiva: Discurso do Sujeito Coletivo. Rev Enferm. 2015; 5 (2): 257 - 266.

Silva J A S, Hinrichsen S L, Brayner A C, Vilella T A S, \& Lemos M C. (2017). Glosas hospitalares e o uso de protocolos assistenciais: revisão integrativa da literatura. Rev Adm Saúde. 2017; 17 (66): 1-18. 
Research, Society and Development, v. 10, n. 10, e229101018684, 2021

(CC BY 4.0) | ISSN 2525-3409 | DOI: http://dx.doi.org/10.33448/rsd-v10i10.18684

Wiley K E, Steffens M, Berry N, Leasky J. (2017). An audit of the quality of online immunization information available to Australian parents. BMC Public Health. 2017; 17 (76): 1-9.

Pissaia L F, Costa A E K C, Moreschi C. \& Rempel C. (2016). Sistematização da enfermagem: impacto da informática e os desafios na qualidade da assistência. Ver Saúde Com. 2016; 12 (4): 737-743.

Instefjord M H, Askjaer K, Espehaug B, \& Graverholt B. (2014). Assessment of quality in psychiatric nursing documentation - a clinical audit. BMC nursing. 2014; 13 (32): 2-7.

Maia A B B, Barbosa AB, Silva M N P, Bbranco L M G C, Rodrigues L M C, \& Melo T T C. (2017). Technical And Scientific Compilation About Audit And Quality Management: An Integrative Review. Rev Enferm UFPE. 2017; 3 (3):1489-1494. 